\title{
Student autonomy of feedback format in higher education and perceived functional behaviours for academic development
}

\author{
${ }^{1}$ Institute of Education, University of Derby, United Kingdom (ORCID: 0000-0003-0609-467X) \\ ${ }^{2}$ Institute of Education, University of Derby, United Kingdom (ORCID: 0000-0003-2251-0039) \\ ${ }^{3}$ Institute of Education, University of Derby, United Kingdom (ORCID: 0000-0002-3872-2725) \\ ${ }^{4}$ Institute of Education, University of Derby, United Kingdom (ORCID: 0000-0001-8529-050X) \\ ${ }^{5}$ Institute of Education, University of Derby, United Kingdom (ORCID: 0000-0002-1075 -1567) \\ ${ }^{6}$ Institute of Education, University of Derby, United Kingdom (ORCID: 0000-0002-5628-1951)
}

Abby Sparrow ${ }^{1}$, Samantha Smith ${ }^{2}$, Dominic Petronzi ${ }^{3}$, Helen Wilson ${ }^{4}$, Sarah Roeschlaub ${ }^{5}$ and Melanie Smith $^{6}$

\begin{abstract}
In the current context of promoting active learning and raising student engagement within Higher Education, an increasing amount of research has looked at pedagogical-based design and factors that contribute to functional behaviours surrounding the interaction and use of academic assessment feedback. However, few studies have considered the perceived influence of student autonomy over feedback format and whether this promotes engagement and academic development. In this study, we recruited level 5 and 6 students $(N=38)$ on an undergraduate Education Programme (that has consistently implemented student feedback choice) to participate in initial self-reporting and subsequent focus groups ('soft triangulation'). The findings revealed three core themes: [1] Personalisation - (a) sense of autonomy/involvement, (b) engagement and (c) motivation, [2] Clarity - (d) depth and detail, and [3] Areas for development. Overall, these findings suggest that feedback type - and the inherent option to choose - has a functional impact on academic engagement and development. We discuss these findings in relation to a sense of being valued that was associated with autonomy of choice, a divergence in how and when students engage with feedback, as well as the requirement for academic clarity and provision of formats that support academic development.
\end{abstract}

Keywords: Feedback; Feedback choice; Active learning; Academic development; Pedagogy

Article History: Submitted 7 March 2020; Revised 13 April 2020; Published online 11 May 2020

\section{Introduction}

It is commonly agreed that feedback for university assignments is crucial for students' academic development, yet the benefits of varied feedback formats is notably under researched (FernandezToro \& Furnborough 2014; King, 2015; Winstone, Nashb, Rowntreea, \& Menezesa, 2016). Existing literature has often identified key facets relating to assessment feedback, including [1] the level of clarification and detail in which to aid student understanding of feedback, and [2] the importance of a common understanding between tutor and student in regard to the nature and purpose of

Address of Corresponding Author

Dr. Dominic Petronzi, Institute of Education, University of Derby, Kedleston Road, Derby, DE22 1GB, United Kingdom.

$\triangle$ d.petronzi@derby.ac.uk

How to cite: Sparrow, A., Smith, S., Petronzi, D., Wilson, H., Roeschlaub, S., \& Smith, M. (2020). Student autonomy of feedback format in higher education and perceived functional behaviours for academic development. Journal of Pedagogical Research, 4(2), 98-111. 
feedback. Literature in this area appears to be generally concerned with achieving the best outcomes for students. However, the common - and current - practice of digitised feedback has been criticised for its perceived distance and limited scope for clarification (Electronic Feedback Survey, 2010, as cited by Budge, 2011). Thus, this research centred on synthesising feedback-based projects to support the wider exploration of core elements that contribute to greater student engagement and academic development. Although focusing on a UK perspective, a brief acknowledgement of international approaches to feedback shows consistency with traditional formats that are potentially limited in satisfying or supporting students academically (Al-Bashir, Kabir \& Rahman, 2016) suggesting a universal area for development within education. Indeed, with a move towards Personal Academic Tutoring across UK Higher Education Institutions (HEIs), a student's selection of a preferred feedback format may provide a more tailored, informative and autonomous review to support academic development. This was a central consideration of the research project, particularly in terms of affective feedback potentially relating to positive self-esteem and motivation.

The requirement for personalised feedback is key, particularly when considering Deci's (1980) Self-Determination Theory (SDT) which emphasises a student psychological need for relatedness in order to become intrinsically motivated and engaged. Deci and Ryan (2000) further posited that students should feel a degree of care and warmth from their tutor. However, with a number of HEIs adopting anonymised feedback - while intended to minimise implicit bias (Baird, 1998) may also be regarded as counterproductive with regards to personalisation. Indeed, the process of anonymization has not been systematically explored, although survey data collected with UK undergraduate students (Pitt \& Winstone, 2018) indicated that there was no significant difference in grades given for anonymous and named assessments. However, the results suggest that anonymous marking may undermine the learning potential of feedback and minimise the strength of the relationship between lecturers and students (Pitt \& Winstone, 2018). Notwithstanding, the anonymous marking process has been theorised to protect the integrity of an academic and student relationship (Malouff, Emmerton \& Schutte, 2013) although at a potential cost of personalisation. Thus, the clear disparity in findings - and generally limited evidence necessitates further enquiry and was a peripheral focus of this research. At the time of writing, anonymous marking had been in position for a single semester.

Although based on online educational courses, a number of factors have been found to have associations with learner retention, including: [1] the extent of access learners have with the course academic, [2] course resources and peer interaction (Marks, Sibley, \& Arbaugh, 2005; Hew, 2016) and, crucially, [3] academic interaction (interpersonal skills) with learners (Hone \& El Said, 2016). Grieve, Moffitt and Padgett (2019) believe that there is a perceived social distance between tutors and students that must be eliminated to encourage approachability and guidance seeking behaviours. Indeed, Skipper and Douglas (2015) acknowledged the potential impact of feedback upon learning, achievement and tutor-student relationships, with an overall association with academic outcomes - both positive and negative. Aligning with evidence in this area, the current research endeavoured to explore student perceptions of feedback and whether this supports academic development. A focal point of this project was to determine whether the practice of allowing feedback type choice of: [1] in-text comments, [2] a detailed feedback summary, [3] audio, or [4] tutorial based, throughout an undergraduate degree (Child \& Youth Studies; BA) had supported academic development for level 5 (stage 2) and 6 (stage 3) students (UK HE third year) at UK university. The feedback offered addresses 6 core areas. Consistent with the availability of audio feedback on the course, Rawle, Thuna, Zhao and Kaler (2018) explored student perspectives of this format as an alternative to traditional written feedback. The study utilised qualitative methodology through open-ended surveys and interviews with 6 teaching assistants and 821 students in a Higher Education biology programme. The findings suggested that audio feedback was considerably more personal and had encouraged students to engage more with the feedback. Moreover, students were appreciative of the level of feedback offered through audio formats and 
was found to improve understanding. However, students alluded to occasional difficulty in identifying which section of the work the audio was referring to, leading to some confusion. To address this limitation, Cullen's (2011) empirical work had previously indicated that in-text written comments, alongside audio feedback are necessary to improve clarity and establish the intended interpretation. The choice of audio feedback for students on the Child and Youth Studies (CYS) programme was explored in this research to further shed light on perceptions of this format. Similarly - although relating to written feedback - Sopina and McNeill (2015) found from surveys of 335 students on a Higher Education health course that more encouragement was reported when the feedback tone and language used by tutors was more positive. Consistent with this finding, the written feedback (and audio/face-to-face discussions) offered through the CYS course is constructive, supportive and emphasises strengths surrounding 6 main assessment criteria, regardless of the type of feedback chosen by the student.

Within feedback-based research, clarity has been identified as another core element that also increases satisfaction (Sopina \& McNeill, 2015). Manning (2013) points to inadequate feedback (lack of clarity) as having an adverse effect on student well-being in terms of heightened levels of stress and reduced intrinsic motivation. Indeed, Al-Bashir et al., (2016) emphasise that when a student better understands their goals and expectations, a route of progression is more clearly identified. According to additional (and more recent) research in the area (e.g. Ajjawi \& Boud, 2015; Dawson, Henderson, Mahoney, Phillips, Ryan, \& Boud, 2019) clarity of feedback - and in the case of CYS, feedforward points - has been shown to motivate, increase learning and understanding, and encourage reflection in students - a key attribute in the process of continuous learning, growth and development. Building on the notion of learning and understanding, Green (2019) explored the perceptions of feedback - albeit with only a single international student studying in a UK HEI. Thus, although findings are subject to methodological limitations, Green concluded that feedback - in a general sense - is of little value if it is based on transmission; essentially, a one-way process and contradicts the movement towards active interaction. A lack of engagement with feedback - for various reasons - can impact subsequent attainment. Specifically, ineffective feedback (and communication of this) may induce pessimistic attitudes and contribute towards a lower self-efficacy (Zimmerman, 2000) that can lead to motivational and cognitive deficits (Kolacinski, 2003). Self-Efficacy Theory relates to social cognitive theory (Bandura, 1986) and is based on the premise that individuals will more likely engage in activities if they believe they have the capability to complete them. Relating to this, individuals often consider the difficulty level of the task and evaluate their belief of succeeding. As self-efficacy can be an outcomedependent factor in a wide-range of activities, it is therefore essential that feedback is meaningful and applicable for the students to encourage their development and engagements. This may require a diversion from traditional feedback formats and those considered most effective by academics. This is again explored in this research in accordance with an approach adopted on a programme at a UK university. 'Quality feedback' can be regarded as multi-faceted, with interpersonal skills of tutors having a principal influence on the 'student journey' (Brodie \& Jolly, 2012). Indeed, this facet of the academic skillset is more pronounced and vital in the current Personal Academic Tutoring process within UK HEIs, although more specifically, the tutorialbased feedback option offered on the CYS course allows a student to have meaningful discussions with the module tutor whom marked their work - contributing to clarity. Further supporting this approach, London (2015) and King (2015) both regarded feedback-based discussions as allowing for more clarity to be explored, as well as ensuring correct student interpretation. This aligns with the nature and purpose of feedback.

While the perception of feedback has substantially altered and developed with time, purposeful feedback and approaches to this has been susceptible to misunderstanding, both from educators and students (Dawson et al., 2019). Indeed, misalignment of educator/student perceptions of feedback can result in a less effective process (Orsmond \& Merry 2011). Aligning with the CYS programme, Orsmond and Merry (2011) also suggest that scaffolding and variations in feedback 
styles that are tailored towards student preference have a greater impact on learning, and requires educators to exercise a set of skilful pedagogical skills to understand students' understanding and ascertain their needs and readiness for appropriate feedback. However, Chi, Siler and Jeong (2004) previously cautioned against tutor dominance within the feedback process to prevent this becoming a logical process that simply follows curriculum guidelines. Adding to this, Green (2019) considers the limited effectiveness of written feedback and posits that audio and face-to-face tutorials enhance the effectiveness and measuring of engagement and understanding through dialogue that encourages information seeking behaviours - as opposed to a tutor directed conversation. However, this is dependent on student preferences. Indeed, evidence has also shown that students often show preference for the feedback style that they are accustomed - but is not necessarily the most ideal for them (Jones, Yeoman, Gaskell \& Prendergast, 2017). Hence, the CYS programme provides students with all types of feedback initially, allowing a more informed choice for subsequent module feedback.

In sum, research evidence indicates that a range of factors, including tutor personality, clarification, and mutual understanding of feedback all have significant impacts on student engagement with the feedback process. Discouragingly, feedback has become a practice that many students do not engage with as intended, and often only seek to obtain their awarded grade with little concern for the advice offered to support their academic development (Carless, 2006). In light of this - and to explore perceptions of the CYS programme approach to assessment feedback - the research aimed to explore experience and feedback formats: [1] regarding student engagement with their chosen feedback style, and [2] whether there was a perceived impact on student approaches to subsequent assignments. The research objectives were: [1] to initially obtain insight through a short self-report questionnaire, [2] that subsequently informed and contributed towards focus group discussions. Specifically, the research endeavoured to explore whether a choice of feedback type is regarded as valuable for subsequent learning and academic development.

\section{Method}

\subsection{Research Design}

The research design was twofold: [1] a self-report quantitative instrument (with open feedback sections) [2] that supported questioning across focus group. For this research, we refer to Morse (1994) who considered qualitative research as the heart of important work, hence main student feedback was sought through this methodology.

\subsection{Analytic Design}

The core research findings were obtained through focus groups with level 5 and 6 students whom elaborated on their undergraduate feedback experiences. Focus group data was analysed using Thematic Analysis (TA) and adhered to the guidelines of Braun and Clarke (2006). Thematic analysis was considered as favourable in light of additional analytic approaches, for example, Interpretative Phenomenological Analysis (IPA) as TA is useful for gaining initial insight and knowledge to form broader patterns for subsequent research and analysis (Smith \& Osborn, 2003), aligning with the pilot study approach of this project in providing the foundations for subsequent in-depth inquiry. Similarly, While IPA may be used for later research in this area that considers participant insight at a much greater depth - as is considered preferable (Hefferon \& GliRodriguez, 2011) - this student supported research - in accordance with the principles of an Undergraduate Research Scholarship Scheme (URSS) - benefitted from a more small-scale and inductive exploration.

Thematic analysis allowed for exploration of the factors associated with student experiences of the CYS programme feedback approach, as well as considering perceptions of how these impacted academic development throughout an undergraduate degree. This enabled identification of pertinent themes in accordance with thematic analysis principles, and provided perceptions of how feedback has been utilised; what specific aspects have been supportive - as well as limited - 
and how the experience of the feedback approach has characterised and influenced academic behaviours and attitudes (Berg, 2004). The research employed an inductive approach by allowing the data to naturally inform, rather than imposing preconceived categories or frameworks (Moretti et al., 2011).

\subsection{Participants}

Undergraduate students within the Institute of Education (level 5 \& 6) at a UK university in the East Midlands region took part in the research process. All participants had previous experiences of the option to choose experience and so met inclusion criteria. Specifically, a convenience sample gave access to Child and Youth Studies (BA) students to complete the self-report questionnaire $(N=38)$. Following collection and analysis of this data (frequencies) focus groups were formed with level $5(N=7 ; 6$ females \& 1 male) and level 6 students $(N=8$; female-based).

\subsection{Instruments}

\subsubsection{Perceptions of Feedback and Impact Questionnaire (PFIQ)}

The formulation of the PFIQ drew on literature and academic knowledge, undergoing a process of item redundancy with 4 academics within the Institute of Education whom had previous experience of offering choice in feedback format. To increase content-validity, items were checked for terminology, potential bias, and to ensure relevancy and precision in terms of informing some additional focus group questions. This process lead to a number of terminology-based amendments, and some items were dropped based on similarity. The refined instrument consisted of items that considered feedback effectiveness, academic development benefit and personal engagement (see Table 1$)$ using a Likert-scale response format $(1=$ completely disagree; $5=$ completely agree). For other items, participants were specifically asked to identify a type of available feedback on their course (by selecting one of the provided feedback formats presented as a Likert-scale) for example, "which type of feedback is most effective in helping you to understand where or how improvement is needed?" The PFIQ also included open ended opportunities, allowing participants to provide optional and additional perspective that may not have been addressed by the PFIQ items.

Table 1

Dimensions of the PFIQ and sample items

\begin{tabular}{ll}
\hline $\begin{array}{l}\text { Feedback } \\
\text { Effectiveness }\end{array}$ & Sample Items \\
\hline $\begin{array}{l}\text { Academic } \\
\begin{array}{l}\text { Development } \\
\text { Benefit }\end{array}\end{array}$ & $\begin{array}{l}\text { Item 3: I find that feedback helps me to improve future work. } \\
\text { assignments. }\end{array}$ \\
\hline $\begin{array}{l}\text { Personal } \\
\text { Engagement }\end{array}$ & $\begin{array}{l}\text { Item 5: It helps to engage with feedback when I come to write my next assignment. } \\
\text { Item 4: It helps to listen to engage with feedback presented to me as soon as I receive } \\
\text { it. }\end{array}$ \\
\hline
\end{tabular}

\subsubsection{Focus Groups (following PFIQ)}

In addition to 7 standardised semi-structured questions (see Table 2) that were framed around literature in the area, PFIQ responses were drawn on generally for additional questioning across the focus groups (level 5, $N=7$; level 6, N=8;) in accordance with 'soft' methodological triangulation (Turner \& Turner, 2009). 
Table 2

Focus Group standardised questions

Sample Focus Group Questions

Q1: Do you feel that feedback for your assignments helps you to improve your work in the future? If so, how?

Q2: Do you feel that you fully understand the feedback that you receive? E.g. language used by the tutor or advice given for how to improve.

Q3: When do you usually engage with your feedback? In what way does this help you with your assignments?

\subsection{Procedure}

Level 5 and 6 students that were available (due to lecture, placement and other commitments; convenience sampling) first completed the PFIQ. Following this, initial analysis was completed to ensure that feedback preference perspectives were captured in additional questions. Subsequently, focus groups were formulated through convenience sampling, and upon completion, data was transcribed and analysed in accordance with TA principles following an inductive approach.

\subsection{Data Analysis}

The analysis process followed the 6 stipulated stages (Braun \& Clarke, 2006). The guidelines urge [1] familiarisation with the data and a detailed understanding of the content; [2] which then enables an initial list of codes to be produced. [3] Themes can then be generated through refocusing the analysis and sorting codes [4] that are then refined with some becoming redundant. This leaves themes that best encapsulate the data, though further refinement may be required. [6] At this phase, a full set of themes has been established and data is reported. Adherence to this process lead to the findings of 3 core themes and associated sub-themes that captured the discussions in full.

\subsection{Ethics}

The research was cleared through the university Research Ethics Committee and adhered to the British Psychological Society ethical guidelines.

\section{Results and Discussion}

This analysis presents direct accounts of factors that final year undergraduate students (UK level 5 \& 6) discussed as core feedback types/preferences and the impact of these. The open feedback was examined in relation to the thoughts and experiences of learners. Initial themes were extracted due to their importance and restructuring. In accordance with the inductive thematic concept that discourse generates knowledge, emerging codes deemed as important and significantly representing perceptions of feedback were categorised and developed until three main themes and associated subthemes were identified as capturing the discussions in their entirety: [1] Personalisation - (a) sense of autonomy/involvement, (b) engagement and (c) motivation, [2] Clarity - (d) depth and detail, and [3] Areas for development. This is represented in figure 1, as are frequencies from the Perceptions of Feedback and Impact Questionnaire, showing the feedback types students typically opted for, as well as those they considered to be the most useful. The feedback options available to students ultimately relate to the core themes and subthemes that emerged from the focus groups. 


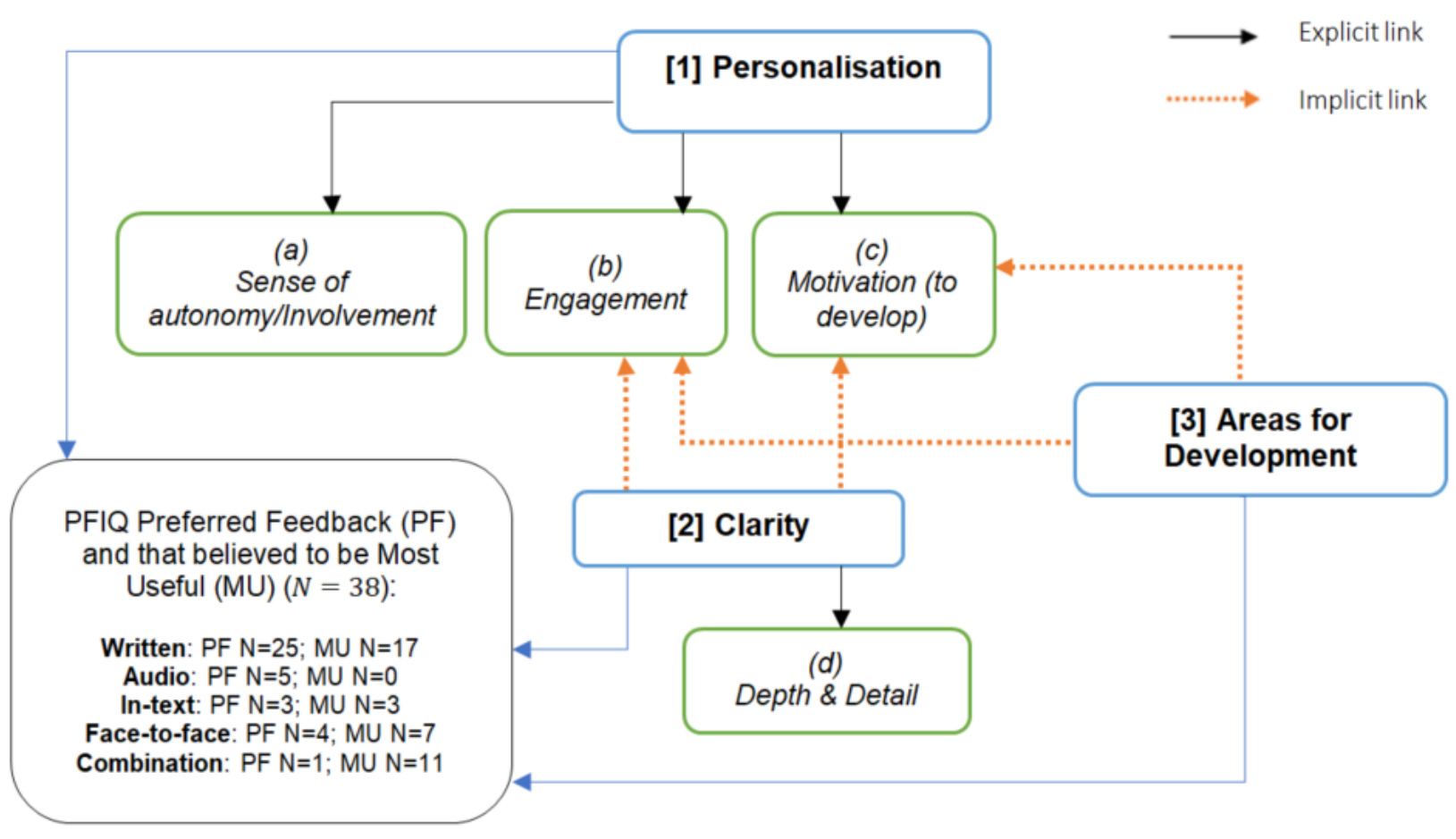

Figure 1. Thematic map of the core themes identified in discussions with level 5 and 6 students

\subsection{Theme 1 - Personalisation - sense of autonomy/involvement, engagement and motivation}

Personalisation was naturally identified as a pertinent theme as this was core to the research discussion. However, as part of these conversations, students revealed how feedback choice supports their engagement and motivation to work towards this feedback for subsequent assessments - linking to their academic development - as well as highlighting the importance of clarity of feedback. However, subsequent core themes of 'Clarity' and 'Areas for Development' also - but more implicitly - linked to subthemes of (b) engagement and (c) motivation (see figure 1).

The focus groups revealed that students generally perceived the CYS modules and programme to be "tailored to needs" when given the opportunity to independently select assessment feedback type. Interestingly, there was discussion surrounding a sense of being "valued" by academics, which created a sense of belonging and closer working relationship with the lecturers. Moreover, students indicated that choice contributed to their learning and development.

"A choice of feedback makes us feel valued and closer to the academics" - P1 (lvl 6).

"Personalised feedback provides a more intimate view and allows you to feel more involved in the marking process" - P8 (lvl 6).

"It gives us some control over what we want and what works for us" - P3 (lvl 5).

"...Because we have the choice, we're probably more involved than other students on other courses might be" - P6 (lvl 5).

This - and the extracted discussions points - supports Grieve et al., (2019) who emphasise that distance between tutors and students can prevent students feeling comfortable to seek guidance and may result in poorer academic performance. In accordance with Deci's (1980) SelfDetermination Theory (SDT; a sense of relatedness increasing intrinsic motivation and engagement) students indicated that personalised feedback - in accordance with their freedom of choice - gave them a greater sense of being a part of the marking and feedback process. Inclusion and autonomy of choice is posited to minimise transmission-based feedback and increase the value of feedback (Green, 2019). A pertinent point raised - and more widely supported in discussions - 
centred on motivation induced by relevant and meaningful feedback that initially stems from personal preference.

"A choice of feedback type gives makes me actually read the feedback and then try to improve" - P5 (lvl 5).

While this aligns with Al-Bashir et al., (2016) who emphasised that when a student better understands their goals and expectations, a route of progression becomes more obvious, it also gives further insight regarding interaction with feedback. This comment suggested that feedback even if detailed and useful for academic development - may not be considered (read) by the student if they do not have a sense of ownership over this - which is seemingly what feedback choice promotes. When this was explored further, other students elaborated on this:

"You're more likely to engage with it [feedback] if it's what you wanted" - P3 (lvl 5).

“... if you get audio and you didn't ask for it and don't like it, you just won't listen to it" - P4 (lvl 5).

These comments further support the perspective that students may engage less with standard feedback approaches e.g. in-text comments across the board, and therefore, choice of feedback type seems to link with motivation to: [1] engage with the feedback, and [2] attempt to apply the feedback to support academic development. Although some students may only seek their grade with little concern for the offered advice (Carless, 2006), offering a choice of feedback is perhaps a method that increases engagement. All learners have different needs and preferences, and this was evident in some identifying in-text comments as their ideal feedback format due to the detail given and the intuitive nature of this format.

"If it's personalised, I engage with it and feel motivated to improve for next time" - P5 (lvl 5).

"In-text allows for precision and examples of rectified work" - P3 (lvl 5).

Cullen (2011) previously indicated that in-text written comments alongside audio feedback are necessary to improve clarity and establish the intended interpretation. Whilst written feedback was the preferred format (figure 1) students believed that this should be in conjunction with a follow-up meeting for clarity and depth. This further serves to demonstrate the variation in student feedback type preference and supports this as a more widely adopted approach to meet a diverse range of needs. Of particular interest was a discussion surrounding feedback choice as supporting independent identification of common areas for development - perhaps due to their increased engagement with this:

"When feedback is what you wanted, it helps identify common themes in your academic writing" - P1 (lvl 5).

Independent feedback choice - and providing a range of formats - allows a student to be more actively engaged in their learning and development, perhaps through creating a sense of ownership and responsibility.

\subsection{Theme 2 - Clarity}

In accordance with existing feedback-based research, students discussed the importance of clarity and the impact of this on understanding and engagement. Al-Bashir et al., (2016) posited that there is increased understanding, goal, expectations and a route to progression when feedback is clear which has also been shown to motivate and encourage reflection (Ajjawi and Boud, 2015; Dawson, et al., 2019).

"Elaborated feedback is very helpful. It's helpful to have more of a clear explanation about specific improvements" - P6 (lvl 5).

"If it [feedback] is detailed but makes sense, I know what I need to do for next time, and it means I can be quite confident in improving" - P6 (lvl 5). 
As part of the CYS Programme marking - which continues to trial new approaches - a simple but seemingly well received amendment to feedback centred on introducing a marking approach that maintained focus on 6 key criteria, but additionally highlighted key examples and amended accordingly. A sample of the feedback communication provided to students is shown below:

"Some key examples of your work have been picked out and amended in the 'feedback summary' tab to show you how this could be improved as you move through your studies. Some other example points have been highlighted in yellow text and numbered according to the criteria they match, whereas areas of strength are highlighted in green text.'

In response to this feedback approach, students discussed satisfaction, as it not only clearly demonstrates exemplar academic writing, but also shows areas of good practice and areas for development. Referring to previous comments surrounding the quality of feedback as being essential in determining whether students actually read and subsequently apply this, students indicated that this approach would support their development:

"6 clear and consistent criteria help me to know specifically where to improve and focus my efforts" P6 (lvl 5).

"Highlighting green and yellow makes it much clearer and obvious" - P2 (lvl 5).

"It's nice to see clearly identified examples of what I've done well. It will be easier to go back to it" P7 (lvl 5).

Discussions with students also indicated that whilst written feedback in accordance with 6 key academic criteria was beneficial for development, there was increased impact - and clarity - when feedback was followed by a face-to-face meeting, as this presumably promotes personalisation. In accordance with London (2015) and King (2015), feedback-based discussions can further contribute towards increased clarity. Notably, a combination of feedback was shown by the frequencies $(\mathrm{N}=11)$ to be considered as the most useful approach. This is an approach that, while implicitly supported by the PAT process following a marking period, could be encouraged and made more explicit. Indeed, a student felt that, "... a face to face meeting should be made mandatory." This further aligns with Green's (2019) more recent emphasis on two-way communication and dialogue as pivotal in student understanding of academic work and areas for development. This was seemingly reinforced by some students in the focus groups:

"Written [feedback] is good because you can go back to it later on, but it isn't in-depth enough" - P3 (lvl 6).

"It [written feedback] does well, but it needs to be followed by a meeting for clarity" - P1 \{supported in level 5 discussions\} (lvl 6).

Furthermore - and in support of Rawle et al., (2018) students suggested that audio feedback may sometimes be the only format that is simple to understand and refer back to, while others considered this to be a potentially beneficial format, although interestingly, felt that it should not be time limited (currently timed out at 3 minutes). Of further interest are the PFIQ based frequencies that show while students do opt for this format, overall, frequencies show that it was not considered to be the most useful type of feedback (Figure 1).

"I have chosen audio before because I don't understand the [written] feedback when I look at it at a later point" - P7 (lvl 5).

"I don't choose it [audio] because it tends to be quite fast and I need more time" - P2 (lvl 5).

As a final point to consider, a student stated that clarity was essential from their perspective as they did not consider or engage with feedback until nearer the next assessment period. Therefore, they required that feedback was clear to support their later engagement with this. Additionally, from an academic perspective, if feedback is clear, this will support their ability to more effectively 
refer back to this if asked to by the student. This short discussion was deemed important as it further reveals the varying feedback interaction approaches of students, and the importance of feedback that can be drawn on at any time, not just in the immediate post assessment period. Indeed, there are digital formats that may support storage and amalgamation of feedback, although it remains that if not inherently detailed and clear, student engagement, motivation and development may be adversely impacted.

\subsection{Theme 3 - Areas for development in marking}

While students discussed strengths of the marking process adopted by the CYS programme, they also indicated further directions for development that could be adopted across programmes and HEIs. Students felt that they were not always entirely sure what to expect with regards to each feedback type, and thus felt that standardised directions - that aligns with anonymous marking procedures - could be provided for each assessment that allowed them to make a more informed decision with supporting examples. Crucially, frequencies indicate that personal preference does not necessarily equate to most useful and reaffirms that format choice may be based solely on routine, rather than beneficence (Jones et al., 2017) and necessitates support in making an informed decision.

"Examples of what the choices would be like in the marking before choosing - this could be on a standardised front cover provided for students for each assessment" - P1 (lvl 5).

"Yes [in response to P1] that would really help me because I can't always remember what each format is like" P2 (lol 5).

Students also made clear that feedback has to have meaningful and actionable points, otherwise they felt that it would be of little relevance to them.

"Feedback isn't helpful when there isn't much to understand" - P7 (lvl 6).

"Feedback is not written in a way to help me improve, for example 'awkward phrasing' - "I do not get how it's awkward, so I just disregard it" - P8 (lol 6).

Whilst some students believe that anonymous marking was a progressive strategy and would eliminate potential bias within grading:

"I like the fact that I am just a number, it will disqualify potential bias" - P1 (lvl 6) \{supported by P3, P6\}.

Winstone (2018) previously found no difference in grades given, irrespective of personalised or anonymous assignments. With regard to anonymous marking, convergence was found across the year groups and was more favoured by level 5 students. Concerns were raised within open ended questions on PFIQ that anonymous feedback would impact student involvement in the marking process and potentially remove a degree of personalisation. However, further research surrounding this approach to marking and the impact on student engagement with feedback is required. Notwithstanding, our current findings indicate that this is a process that has little impact when choice of feedback is offered, and there is clarity and direction for academic development.

\section{Conclusions}

The current study explored student perspectives surrounding assessment feedback choice of a non-institutional wide approach and the broader implications of this. Crucially, discussions addressed criteria often outlined in previous research in terms of personalisation (Green, 2019; Grieve et al., 2019) and clarity (see e.g. Manning, 2013; Sopina \& Mcneill, 2015) while students also indicated areas for subsequent improvement that focused on more detailed exemplars of each available feedback format. Specifically, discussions illuminated student behaviours and thought processes that may be determinants of academic engagement and attainment. Overall, these findings suggest that feedback type - and the inherent option to choose - has a functional impact 
on academic engagement and development. The data that we have presented lead to a number of important implications, not only for how academics approach the feedback process and the available provision, but also for the student experience and how this can influence engagement. These implications are now considered:

\subsection{Autonomy of Feedback Format}

Despite literature indicating several features surrounding feedback that may contribute to more functional academic behaviours, including interaction with and interpersonal skills of the academic (Hone \& El Said, 2016), phrasing and terminology (Manning, 2013) and type of feedback e.g. written and audio (Cullen, 2011; Rawle et al., 2018), there has been limited emphasis on autonomous choice and the perceived impact of this. While Orsmond and Merry (2011) considered varied feedback styles and student preference, our findings centre on students accustomed to these embedded pedagogical approaches. Students felt that a choice of formats tailored the feedback process to their individual needs and lead to a sense of control and more functional behaviours that align with a movement towards active learning. Students also revealed a dynamic approach to utilising effective feedback in terms of how and when they refer back to this - with minimised options to use feedback if regarded as unsupportive of development. It seems effective feedback remains valuable beyond assessment periods and was a key requirement for some who discussed a preference towards engaging with feedback later into the subsequent semester. Equally, clarity of feedback reinforces academic ability to refer back to this - if requested by the student - post assessment period. Thus, we suggest that effective feedback can be regarded as live and active, and not merely to justify a current grade. As a caveat to this point, our findings support literature (see e.g. Carless, 2006) that suggests detailed feedback may be disregarded if students do not sense a degree of ownership. Indeed, discussions gave some insight surrounding feedback engagement behaviours in terms of a process of first reading and then (deriving from this) acknowledging/applying this, which was framed around the initial autonomous choice of preferred format.

\subsection{Importance of Feedback Clarity}

A comprehensible route for academic development was deliberated by students, and clarity of feedback (Ajjawi \& Boud, 2015; Dawson et al., 2019) was indicated to be a facilitative factor of motivation to improve and increase understanding (Al-Bashir et al., 2016). Convoluted phrasing was suggested as adversely impacting understanding and discussions surrounding the ability to improve can be framed around self-efficacy (Zimmerman, 2000) as an associate of motivation and cognitive deficits (Kolacinski, 2003). Our findings revealed that a more systematic feedback approach adopted by the CYS programme (the addition of colour coding and amending specific examples within written feedback) was well received by students who suggested this was more intuitive and allowed for ease of reference at a later stage. Moreover, and similar to London (2015) and King (2015), follow up discussions with the marking tutor were suggested as being important for additional understanding of feedback and places emphasis on the interpersonal skills of academics (Hone \& El Said, 2016).

\subsection{Feedback Formants}

While PFIQ frequencies show that written feedback was the most favoured approach across the participants, this did not necessarily equate to being perceived as the most beneficial (figure 1). Thus, our findings seem to support previous suggestions that students - if given a choice - opt for what they are accustomed to, rather than what is ideal (Jones et al., 2017). It may be that previous educational experiences, particularly a lack of autonomy, contribute to increased preference for more traditional written feedback. Subsequent discussions focused on perceived areas for development, and most notably, support was expressed for various feedback type exemplars as part of a standardised assessment submission cover to enable more informed decisions. Indeed, this is first dependent on a range of feedback formats being made available. Divergence was also 
noted surrounding perceptions of audio feedback that was shown by the PFIQ frequencies to be a less favoured choice than others. Some felt that audio may enable ease of reference at later stages, whereas some referred to this being time limited - too detailed information in a short amount of time (software determined). This again shows key differences in student feedback preferences and highlights the importance of choice being embedded within Higher Education course provision to meet a range of needs and approaches.

\section{Limitations and Future Direction}

The results of this study are not without limitation. First, we acknowledge the inherent methodological limitations surrounding subjectivity associated with qualitative based research, in addition to a restricted demographic and perspectives that represent a single undergraduate course at one Higher Education Institution within the UK. Similarly, the participating students are accustomed to selecting preferred feedback, and thus, it would be of interest to see if convergence of perspective is found in research with students that currently obtain feedback in more traditional formats and are not afforded format options.

We are mindful that data was collected in the academic year (2019-20) and still within the adjusting period with regards to anonymous marking which was implemented in the Autumn semester (2019). Thus, student perceptions may - to a degree - reflect more state-based judgements that arguably require further experience of this approach to allow for more informed verdicts.

Further to this, while the Perceptions of Feedback and Impact Questionnaire was created and implemented to support 'soft' methodological triangulation, it would benefit from validation and psychometric testing to ensure that this is a reliable measure of judgements of feedback approaches. This would require amendment to reflect a purely Likert-based measure that maintains the current scoring $(1=$ completely disagree; $5=$ completely agree) of 8 -items in accordance with factor analysis criteria e.g. $>.512$ for $\mathrm{N}>100$ (Stevens, 1992, as cited in Field, 2009).

At the university, Digital Badges (DBs) have become an integral part of our Massive Open Online Courses (MOOCs) as part of our online provision, with an initial observed statistical increase in engagement (Petronzi \& Hadi, 2017). These have been adopted to a degree for purposes of staff training and skills auditing. However, DBs could be more widely implemented to showcase student engagement and accomplishment with potential feedback related tasks (that require a degree of evidence) to support student interaction and engagement, that could ultimately contribute to academic development. This is perhaps an awardable formative measure of student comprehension of academic assessment feedback. To make these more meaningful, DBs might be separated into levels to represent continuous development throughout an undergraduate course e.g. critical evaluation level 4, level 5 and level 6 (potentially level 7 if continuing to postgraduate). This may allow students to obtain greater acknowledgement of a wider range of achievements and skills (Graduate Attributes) and enhance employability prospects.

Finally, at the time of writing, we are in unprecedented times surrounding the challenges faced by the Covid-19 pandemic, and in an academic context, places increased emphasis on the quality and of feedback provision when face-to-face discussions may not be possible. This would be of interest for additional research, as would exploration of online provision during unprecedented times from the perspective of students who had elected for face-to-face teaching and how this impacts feedback provision. Moreover, it would be of benefit to obtain academic perspective surrounding feedback to determine the extent to which this either converges or diverges with student feedback.

This paper has presented course specific feedback regarding the range and impact of feedback choice and preference. We have found that students value autonomy in the feedback process and feel more academically engaged and motivated due to increased personalisation, and diverge in how, and particularly when they engage with this feedback - sometimes post the assessment period. Crucially, our findings show that choice - although not statistically shown - may be 
associated with whether students initially engage with the feedback they receive at a lower level (reading) regardless of its quality, and suggest that self-selected format could be more widely adopted across programmes and institutions to meet a diverse range of needs and educational approaches. Moreover, the clarity of feedback appears essential, and our research found support for an implemented feedback method that utilises colour coding and amended examples to increase ease of understanding and reference. Finally, and based on research findings, we consider whether solutions such as Digital Badges could be embraced as a measurable acknowledgement of student interaction with feedback relating to core academic skills and Graduate Attributes.

\section{Acknowledgments}

This work was kindly supported by the University of Derby Undergraduate Research Scholarship Scheme (URSS).

\section{References}

Ajjawi, R., \& Boud, D. (2017). Researching feedback dialogue: An interactional analysis approach. Assessment $\mathcal{E}$ Evaluation in Higher Education, 42, 252-265. doi: 10.1080/02602938.2015.1102863.

Al-Bashir, M., Kabir, R., \& Rahman, I. (2016). The value and effectiveness of feedback in improving students' learning and professionalizing teaching in higher education. Journal of Education and Practice, 7, 38-41.

Baird, J. A. (1998). What's in a name? Experiments with blind marking in A-Level examinations. Educational Research, 40, 191-202. doi: 10.1080/0013188980400207.

Bandura, A. (1986). Social foundations of thought and action. A social cognitive theory. Englewood Cliffs. NJ: Prentice-Hall.

Berg, B. L. (2004). Qualitative research methods for the social sciences. (5th Ed). Boston: Pearson Education, Inc.

Braun, V., \& Clarke, V. (2006). Using thematic analysis in psychology. Qualitative Research in Psychology, 3, 77-101. doi: 10.1191/1478088706qp063oa.

Brodie, L., \& Jolly, H. (2012). Work in progress: Developing and evaluating tutor training for collaborative teaching," in 2012 IEEE Frontiers in Education Conference (FIE), Seattle, WA, 2012 pp. 1-6. doi: 10.1109/FIE.2012.6462387.

Budge, K. (2011). A desire for the personal: Student perceptions of electronic feedback. International Journal of Teaching and Learning in Higher Education, 23, 342-349.

Carless, D. (2006). Differing perceptions in the feedback process. Studies in Higher Education, 31, 219-233.

Chi, M. T., Siler, S. A., \& Jeong, H. (2004). Can tutors monitor students' understanding accurately? Cognition and instruction, 22, 363-387. doi: 10.1207/s1532690xci2203_4.

Cullen, W. R. (2011) A multi-technology formative assessment strategy. In Middleton, A. (Ed.) Mediaenhanced feedback-Case studies and methods: Papers produced to support the Media-Enhanced Feedback event (pp. 28-33) [Online]. Available at: https://melsig.shu.ac.uk/melsig/files/2017/10/MiddletonMedia-nhanced_feedback_proceedings-final.pdf (Accessed 4th July 2019).

Dawson, P., Henderson, M., Mahoney, P., Phillips, M., Ryan, T. \& Boud, D. (2019) What makes for effective feedback: Staff and student perspectives. Assessment and Evaluation in Higher Education, 44, 25-36. doi: 10.1080/02602938.2018.1467877.

Deci, E. L. (1980) The psychology of self-determination. Free Press, MA: Heath.

Deci, E. L., \& Ryan, R. M. (2000). The" what" and" why" of goal pursuits: Human needs and the selfdetermination of behavior. Psychological inquiry, 11, 227-268.

Fernández-Toro, M., \& Furnborough, C. (2014). Feedback on feedback: Eliciting learners' responses to written feedback through student-generated screencasts. Educational Media International, 51, 35-48.

Field, A. P. (2009). Discovering statistics using SPSS. London, England: Sage Publications.

Green, S. (2019). What students don't make of feedback in higher education: An illustrative study. Journal of English for Academic Purposes, 38, 83-94. doi: 10.1016/j.jeap.2019.01.010

Grieve, R., Moffitt, R. L., \& Padgett, C. R. (2019). Student perceptions of marker personality and intelligence: The effect of emoticons in online assignment feedback. Learning and Individual Differences, 69, 232-238.

Hefferon, K., \& Gil-Rodriguez, E. (2011). Interpretative phenomenological analysis. The Psychologist, 24, 756759. 
Hew, K. F. (2016). Promoting engagement in online courses: What strategies can we learn from three highly rated MOOCS. British Journal of Educational Technology, 47, 320-341. doi: 10.1111/bjet.12235.

Hone, K. S., \& El Said, G, R. (2016). Exploring the factors affecting MOOC retention: A survey study. Computers and Education, 98, 157-168. doi: 10.1016/j.compedu.2016.03.016.

Jones, H., Yeoman, K., Gaskell, E, \& Prendergast, J. (2017). Perceptions of university assessment and feedback among post-16 school pupils. Assessment \& Evaluation in Higher Education, 42, 1233-1246. doi: 10.1080/02602938.2016.1259388.

King, P. (2015). When do students benefit from performance feedback? A test of feedback intervention theory in speaking improvement. Communication Quarterly, 64, 1-15. doi: 10.1080/01463373.2015.1078827.

Kolacinski, J. F. (2003). "Mathematics anxiety and learned helplessness" Dissertations from ProQuest.

London, M. (2015) The power of feedback: giving, seeking, and using feedback for performance improvement. New York: Routledge.

Malouff, J. M., A. J. Emmerton, \& N. S. Schutte. (2013). The risk of a halo bias as a reason to keep students anonymous during grading. Teaching of Psychology, 40, 233-237. doi: 10.1177/0098628313487425.

Manning, P. J. (2013) Understanding the impact of inadequate feedback: A means to reduce law student psychological distress, increase motivation, and improve learning outcomes. Cumberland Law Review, 43, 225-258. doi: 10.2139/ssrn.1967280.

Marks, R. B., Sibley, S. D., \& Arbaugh, J. B. (2005). A structural equation model of predictors for effective online learning. Journal of Management Education, 29, 531-563. doi: 10.1177/1052562904271199.

Moretti, F., van Vliet, L., Bensing, J., Deledda, G., Mazzi, M., Rimondini, M., Zimmermann, C., \& Fletcher, I. (2011). A standardized approach to qualitative content analysis of focus group discussions from different countries. Patient Education and Counseling, 82, 420-428. 10.1016/j.pec.2011.01.005.

Orsmond, P., \& Merry, S. (2011). Feedback alignment: Effective and ineffective links between tutors' and students' understanding of coursework feedback. Assessment and Evaluation in Higher Education, 36, 125136. doi: $10.1080 / 02602930903201651$.

Petronzi, D., \& Hadi, M. (2017). The validation and quality assurance of open badges at the University of Derby. Poceedings of the 14th International ePortfolio and Identity Conference (ePIC) (pp. 1-49). Poitiers, France: ADPIOS.

Pitt, E., \& Winstone, N. (2018). The impact of anonymous marking on students' perceptions of fairness, feedback and relationships with lecturers. Assessment and Evaluation in Higher Education, 43, 1183-1193.

Rawle, F., Thuna, M., Zhao, T., \& Kaler, M. (2018). Audio feedback: Student and teaching assistant perspectives on an alternative mode of feedback for written assignments. The Canadian Journal for the Scholarship of Teaching and Learning, 9, 1-17. doi: 10.5206/cjsotl-rcacea.2018.2.2

Skipper, Y., \& Douglas, K. (2015). The influence of teacher feedback on children's perceptions of studentteacher relationships. British Journal of Educational Psychology, 85, 276-288. doi: 10.1111/bjep.12070.

Sopina, E., \& McNeill, R. (2015) Investigating the relationship between quality, format and delivery of feedback for written assignments in higher education. Assessment $\mathcal{E}$ Evaluation in Higher Education, 40, 666-680. doi: 10.1080/02602938.2014.945072.

Stevens, J. P. (1992). Applied multivariate statistics for the social sciences (2nd Ed). Hillsdale, NJ: Erlbaum.

Turner, P., \& Turner, S. (2009). Triangulation in practice. Virtual Reality, 13, 171-181. doi: 10.1007/s10055-009$0117-2$.

Winstone, N. E., Nash, R. A., Rowntree, J., \& Menezes, R. (2016). What do students want most from written feedback information? Distinguishing necessities from luxuries using a budgeting methodology. Assessment \& Evaluation in Higher Education, 41, 1237-1253. doi: 10.1080/02602938.2015.1075956.

Zimmerman, B. J. (2000). Self-efficacy: An essential motive to learn. Contemporary Educational Psychology, 25, 82-91. doi: 10.1006/ceps.1999.1016. 